\title{
Polyperspektivität in der Medizin: Dilemma oder Chance? Zur Ganzheitlichkeit der Homöopathie Hahnemanns
}

\author{
Josef M. Schmidt \\ Institut für Ethik, Geschichte und Theorie der Medizin, Ludwig-Maximilians-Universität München, München, Deutschland
}

\section{Schlüsselwörter}

Medizingeschichte · Medizintheorie · Polyperspektivität ·

Homöopathie · Jainismus · Samuel Hahnemann · Friedrich Nietzsche ·

Platon · Mythos · Logos · Ethos

\section{Zusammenfassung}

Die von Samuel Hahnemann (1755-1843) begründete Homöopathie lässt sich heute aus den verschiedensten Perspektiven betrachten und beurteilen - bedingt durch interne Differenzierungen, externe kritische Einwände sowie einschlägige Forschungsergebnisse aus fast allen Natur- und Geisteswissenschaften. Die kulturgeschichtlichen Wurzeln der jetzt in allen Bereichen, auch in der Medizin, überbordenden Polyperspektivität liegen im Abendland einerseits im traditionellen monotheistischen Wahrheitsbegriff der jüdisch-christlichen Religion, andererseits in einem seit der Aufklärung sich beschleunigenden Rationalisierungs- und Dekonstruktionsprozess innerhalb der Wissenschaften selbst. Sozioökonomische Auswirkungen der zunehmenden Unsicherheit und Überforderung reichen von Orientierungslosigkeit und Delegitimierung der Wissenschaften bis zur Herrschaft von Zahlen und Algorithmen. Zur Bewältigung dieser Herausforderung bieten sich theoretisch etwa die Philosophie des Jainismus (Anekantavada, Syadvada, Nayavada) oder Friedrich Nietzsches an, praktisch vor allem Platons Staatsphilosophie sowie die christliche Trinitätslehre. Indem auch Hahnemanns HomöopathieKonzeption die Dimensionen Mythos, Logos und Ethos umfasst, bietet sein Ansatz ein seit 200 Jahren bewährtes Paradigma, mit dem Polyperspektivitätsdilemma auf eine praktische, realistische und kreative Weise fertig zu werden.

() 2017 S. Karger GmbH, Freiburg

Überarbeitete Version eines Vortrags, gehalten auf dem 72nd LMHI Homeopathic World Congress 2017 in Leipzig am 16. Juni 2017 in der Kongresshalle am Zoo Leipzig, Deutschland.

\section{Keywords}

History of medicine - Theory of medicine - Polyperspectivity . Homeopathy · Jainism · Samuel Hahnemann · Friedrich Nietzsche . Platon $\cdot$ Mythos $\cdot$ Logos $\cdot$ Ethos

\section{Summary}

Polyperspectivity in Medicine: Dilemma or Chance? The Holism of Hahnemann's Homeopathy

Today, homeopathy, as founded by Samuel Hahnemann (17551843), may be viewed and judged from very different perspectives resulting from internal differentiation, external critical objections, and research findings from nearly all sciences and humanities. Polyperspectivity is currently overabundant in all areas, also in medicine. In the Western world, its cultural-historical roots are, on the one hand, the traditional monotheistic concept of truth in the Jewish-Christian religion and, on the other hand, the accelerating process of rationalisation and deconstruction within the sciences. The socioeconomic impacts of increasing insecurity and excessive demands range from disorientation and delegitimization of sciences to the hegemony of numbers and algorithms. Options to comply with this challenge are theoretically, for instance, the philosophy of Jainism (Anekantavada, Syadvada, Nayavada) or of Friedrich Nietzsche, or practically, above all, Plato's state philosophy and the Christian doctrine of trinity. Since Hahnemann's conception of homeopathy encompasses the dimensions mythos, logos, and ethos as well, his approach provides a paradigm that has proved for 200 years to handle the dilemma of polyperspectivity in a practical, realistic, and creative way.

\section{Einleitung}

Die Spannung zwischen einer liberalen Kultur des Polyperspektivismus in den Geisteswissenschaften und Medien einerseits und bedenklichen Anfängen eines neuen politischen und dogmatischen Rigorismus andererseits lässt sich heute - als Widerspiegelung der gesellschaftlichen, wirtschaftlichen und technologischen Rahmenbedingungen - auch im Bereich der Wissenschaften, spezi-

\section{KARGER}

(C) 2017 S. Karger GmbH, Freiburg

Fax +497614520714 Information@Karger.com www.karger.com
Prof. Dr. med. Dr. phil. Josef M. Schmidt Institut für Ethik, Geschichte und Theorie der Medizin Ludwig-Maximilians-Universität München Lessingstrasse 2, 80336 München, Deutschland josef.m.schmidt@lrz.uni-muenchen.de 
ell der Medizin, wahrnehmen. So ist die Heilkunst (téchne iatriké, ars medica), die - wie ihr Gegenstand, der Mensch - naturgemäss eine Fülle von Perspektiven und Dimensionen umfassen und beachten sollte, in zunehmender Gefahr, von einer dem Wohl des Patienten dienenden Kunst der Unvoreingenommenheit und des Polypragmatismus durch methodische Reduktionen und naturalistische Fehlschlüsse zu einem geschlossenen System gerade opportuner Paradigmen verengt zu werden. Komplementäre Heilmethoden wie etwa die Homöopathie werden seit Längerem - trotz ihres Anspruchs auf Ganzheitlichkeit - von der Mehrzahl der öffentlichen Meinungsmacher nur unter materialistisch-ökonomischen Aspekten wie Wirkstoffgehalt, statistische Signifikanzen und Gebührenziffern diskutiert - als ob es angesichts einer 200-jährigen Diskursgeschichte und interdisziplinärer natur- wie geisteswissenschaftlicher Grundlagenforschung dazu keine weiteren Perspektiven gäbe.

Ein Blick in die facettenreiche Geschichte der Medizin, genauer ein kulturphilosophisch inspirierter Exkurs in die Geschichte der Homöopathie, könnte hilfreich sein, hier den Affirmationshorizont zu erweitern. Die These lautet: Was sich im Folgenden am historischen Beispiel der Homöopathie zeigen lässt, könnte eine lange übersehene umfassendere Perspektive auf die gesellschaftliche Einbettung von Medizin und Wissenschaft eröffnen.

\section{Polyperspektivität am Beispiel der Homöopathie}

Vor 200 Jahren, im Jahre 1817, publizierte Samuel Hahnemann (1755-1843) im Vorwort zu seiner «Reinen Arzneimittellehre», unter dem Titel «Nota bene für meine Rezensenten», einen Satz, der auf den ersten Blick einfach und unmissverständlich aussieht, der von seinen Anhängern auch gerne bis heute zitiert wird, dessen sinnhafte Auslegung aber auch geeignet ist, das Wesen der Medizin auf einer tieferen Bedeutungsebene zu erhellen und aus vergessenen Perspektiven zu betrachten. Der Satz, genauer gesagt ein Imperativ, lautet: «Macht's nach, aber macht's genau und sorgfältig nach!» [1].

Damit, so könnte man meinen und so dachte wohl auch Hahnemann, wäre doch alles gesagt. Immerhin lagen zu der von ihm gerade begründeten Homöopathie, auf die sich der Satz primär bezog, ja detaillierte praktische Anweisungen in seinem 1810 erschienenen «Organon der rationellen Heilkunde» vor [2], dazu eine lateinische Materia medica samt Index [3], die ersten Bände einer deutschen Reinen Arzneimittellehre [4] sowie circa 150 Aufsätze und Monographien, in denen Hahnemann seine medizinischen, aber auch philosophischen, religiösen und ethischen Ansichten seinen Fachkollegen ebenso wie einem breiteren Laienpublikum mitgeteilt hatte [5].
Wenn Jesus zu seinen Jüngern sprach «Folgt mir nach!» (deute opíso mou, Mt 4,19; akoloútei moi, Mt 9,9, Mk 10,21, Lk 9,59, Joh 1,43) [6] und zum Beispiel Paracelsus seinen Lesern zurief «Mir nach!» [7], war dies noch im Sinne einer persönlichen Gefolgschaft gemeint. Mit «Macht es nach» ging es Hahnemann offenbar nicht um sich, sondern um die Sache, hier die Homöopathie. Was das sein soll, war für ihn klar. Aber was war und was ist Homöopathie für die anderen?

Die Rezeptionsgeschichte zeigt, dass es noch zu Hahnemanns Lebzeiten sowohl treue Hahnemannianer gab, die seine schriftlichen und mündlichen Instruktionen streng befolgten, als auch bereits sogenannte kritische Homöopathen, die nicht zu einer 1:1-Übernahme seiner Lehre bereit waren, sondern sich die Freiheit nahmen, Teile davon aus einer anderen, eigenen Perspektive zu betrachten. Konsens bestand in der Regel über das Simile-Prinzip, die Arzneimittelprüfungen an Gesunden und die Verwendung von Einzelmitteln in kleinsten Gaben, doch die Konzepte des Potenzierens sowie der Miasmen oder der Absolutheitsanspruch des späten Hahnemann wurden von vielen kritisch gesehen. Auch in der späteren Geschichte der Homöopathie gab es immer wieder Differenzen innerhalb der Homöopathenschaft hinsichtlich dessen, was wahre Homöopathie sei, etwa hinsichtlich der Fragen Hoch- oder Tiefpotenzen, Organo- oder Psychotropie, Abgrenzung gegenüber der Isopathie, KomplexmittelHomöopathie usw. In der heutigen Zeit scheint es innerhalb der Homöopathie selbstverständlich geworden zu sein, mit einer Vielzahl von Schulen, Richtungen oder Strömungen der Homöopathie zu leben, und in der Tat offerieren zeitgenössische Homöopathen (bzw. solche, die sich dafür halten) ein breites Spektrum von Sichtweisen und Interpretationsangeboten [8].

Wenngleich Hahnemann der Überzeugung war, das, was es laut seinem Imperativ nachzumachen galt, eindeutig und verständlich aufgezeichnet und hinterlassen zu haben, sodass für jeden klar sein sollte, was Homöopathie ist, muss doch festgestellt werden, dass die Homöopathie nach 200 Jahren - zusammen mit dem Zeitgeist - unwiderruflich in der Polyperspektivität angekommen ist [9]. Auf dem Büchermarkt oder in einschlägigen Kursankündigungen sind kaum zwei Lehrbücher bzw. Homöopathen zu finden, die genau denselben Ansatz lehren bzw. praktizieren. Der Hintergrund dürfte vor allem ein sozioökonomischer sein: Im Zuge massiver wirtschaftlicher, gesellschaftlicher, politischer, aber auch wissenschaftlicher und kultureller Veränderungen und Umbrüche wurden in den letzten zwei Jahrhunderten zum einen Traditionen, Autoritäten und Dogmen zunehmend hinterfragt, dekonstruiert und relativiert, zum anderen werden von den modernen Märkten Innovationen, Veränderungen und Neugründungen finanziell belohnt, nicht dagegen das Festhalten zum Beispiel an alten Büchern, Personen und Lehren. 
Nicht nur innerhomöopathisch, auch von extern gibt es eine Vielzahl von Perspektiven, aus denen die Homöopathie betrachtet und beurteilt wird. Kritische Sichtweisen reichen etwa von dem Einwand, dass Hochpotenzen rein rechnerisch ultramolekulare Verdünnungen seien, weshalb Homöopathie eine Scheintherapie sein müsse, bis zur selektiven Interpretation ausgewählter klinischer Studien im Rahmen der Standards einer «evidence-based medicine» oder der neodogmatischen Ansicht, die Homöopathie erfülle nicht die Voraussetzungen einer «science-based medicine» $[10,11]$. Der Homöopathie günstige Perspektiven kommen unter anderem aus der Grundlagenforschung, etwa durch neuere Forschungsergebnisse hinsichtlich Nanopartikel [12-15], Hormesis $[16,17]$, Genexpressivität $[18,19]$, Epigenetik [20] usw. $[21,22]$.

Neben der naturwissenschaftlichen gibt es auch eine breite geisteswissenschaftliche Forschung und entsprechend viele Perspektiven auf die Homöopathie. Aus medizinhistorischer Sicht zum Beispiel ist die Homöopathie die langlebigste und am weitesten verbreitete der Heilmethoden, die in der Umbruchszeit der Deutschen Aufklärung und des Deutschen Idealismus entstanden sind. Aus wissenschaftstheoretischer und philosophischer Sicht erscheint sie als eine phänomenologische, heuristisch-hermeneutische, semiotische, holistische und individualisierende Heilkunst - kognitionsbasiert, ressourcenorientiert, salutogenetisch usw. Aus statistischer und sozioökonomischer Sicht scheint sie, Kosten und Nutzen betreffend, wirksam und sicher zu sein, unverdorben von den bekannten Manipulationen der Grossen in der Pharmaindustrie usw. Aus einer evolutionstheoretischen Sicht lässt sich zeigen, dass die Homöopathie durch ihre Aufsplitterung in verschiedene Richtungen sogar einen Überlebensvorteil hatte, da sich so - je nach den kulturellen Rahmenbedingungen in verschiedenen Ländern - die jeweils anpassungsfähigste Spielart durchsetzen und die Homöopathie sich über die Jahrhunderte und Kontinente weiterverbreiten konnte [23]. Aus ökonomischer Sicht schliesslich verbreitert eine Pluralität von angebotenen Kursen, Büchern und Therapeuten zweifellos den Markt, was mit ein Grund für die wirtschaftliche Prosperität der Homöopathie in den westlichen kapitalistischen Ländern ist.

So steht der heutige Marktteilnehmer vor einer nahezu erdrückenden Vielfalt von Perspektiven, aus denen die Homöopathie wahrgenommen, analysiert und beurteilt werden kann, sodass es geradezu verwegen erscheint, vorauszusetzen, dass jeder unter Homöopathie dasselbe versteht. Gibt es vielleicht «die Homöopathie» gar nicht, sondern vielmehr so viele «Homöopathien», wie es Betrachter bzw. Untersucher gibt?

\section{Die Eine Wahrheit versus Polyperspektivität}

Traditionell beruht die abendländische Wissenschaftsgeschichte, in der auch Hahnemann steht, seit der griechischen Antike auf dem Paradigma, dass es eine Wahrheit gebe, dass etwas entweder wahr oder falsch sei. Seit den Vorsokratikern wurde kontinuierlich versucht, möglichst alles aus einem Prinzip zu erklären. Etwa zur gleichen Zeit, zur Zeit der Babylonischen Gefangenschaft der Israeliten, wurde in der jüdischen Religion das Prinzip des Monotheismus fixiert, wie es seitdem in der Tora bzw. im Alten Testament bis heute tradiert wird. Im Gegensatz zum bis dahin vorherrschenden Polytheismus etwa in der ägyptischen und babylonischen Kultur wurde fortan alles, was dem Volk Israel widerfuhr, auf einen einzigen Gott (Jahwe) bezogen, der zudem auch noch eifersüchtig war bzw. ist [24]. Wenn auch das Christentum kein Monotheismus ist, da die göttliche Trinität etwas grundsätzlich anderes, nämlich einen Beziehungszusammenhang von drei göttlichen Personen darstellt, wurde auch hier der Anspruch auf die eine Wahrheit übernommen, etwa wenn Jesus sagt: «Ich bin ... die Wahrheit» (egó eími ... he alétheia, Joh 14,6) [6] usw. Und so zieht sich durch die gesamte westliche Religions-, Philosophie- und Wissenschaftsgeschichte, bis weit über Hahnemann hinaus, wie ein roter Faden das stets gleiche Mantra: Es gibt nur einen Gott und es gibt nur eine Wahrheit!

Friedrich Nietzsche (1844-1900) war - im Abendland - einer der Ersten, der dieses Konzept der einen für alle gültigen Wahrheit kritisch hinterfragte, ja es genealogisch auf eine Fälschung bzw. eine Erfindung lebensuntüchtiger, zu kurz gekommener, ressentimentbeladener Priester oder Philosophen (Sokrates) zurückführte, die durch ihr Monopol auf die vermeintliche Wahrheit, an die sich jeder zu halten habe, die lebenstüchtigen wohlgeratenen Menschen unterdrücken. Wenn Wahrheit als Lüge enttarnt wird, d.h. als Täuschung im Interesse eigener Herrschaftsabsichten über andere, dann ist auch das Konzept des einen einzigen Gottes nicht mehr zu halten, und so liess Nietzsche seinen Zarathustra verkünden: «Gott ist tot!» [25].

Im Gegensatz zu den meisten seiner Zeitgenossen war Nietzsche gegen Ende des 19. Jahrhunderts wohl bewusst, was diese schreckliche Erkenntnis bedeutete: Wenn der Glaube an den einen Gott und damit der Anspruch auf eine allgemeingültige Wahrheit und allgemeinverbindliche Moral philosophisch nicht mehr zu halten war, dann bedeutete dies das Ende der gesamten abendländischen Kultur, die im Nihilismus versinken würde. In der Tat war Nietzsches Philosophie bahnbrechend für die auf ihn folgenden Strömungen des Relativismus, des Konstruktivismus und der Postmoderne bis hin zur Multifunktionalität, Postfaktizität, Postdemokratie usw. Als Folge davon kann das moderne Subjekt bei seiner Orientierung im Leben und auf den allgegenwärtigen Märkten und Börsen 
nicht mehr auf verbindliche Traditionen oder verantwortliche Institutionen zurückgreifen, sondern sieht sich einer Unzahl von Anbietern von Waren sowie Sinn- und Heilsangeboten ausgeliefert, die angeblich alle nur «sein Bestes» wollen - nämlich sein Geld.

Auf der Suche nach Ansätzen und Lösungen des dringlichen Problems, wie mit der heute ubiquitären Polyperspektivität umzugehen sei, kann man allerdings auch schon im alten Indien fündig werden. Etwa 2500 Jahre vor Nietzsche lebt dort, im 6. Jahrhundert v. Chr., Mahávíra, was so viel wie «grosser Held» bedeutet, der Begründer des Jainismus. Er war Zeitgenosse nicht nur des Gautama Buddha, des Begründers des Buddhismus, sondern auch der griechischen Vorsokratiker, möglicherweise auch des persischen Zoroaster und der altjüdischen Propheten zur Zeit des babylonischen Exils. Im Gegensatz zu den strukturell gewalttätigen westlichen und nahöstlichen Konzepten des einen eifersüchtigen Gottes und der einen von allen zu befolgenden Wahrheit lehrte Mahávíra zeitgleich in Indien eine Art Religion bzw. besser einen Lebensweg der Gewaltlosigkeit (ahimsa) gegenüber allem Lebendigen, der letztlich zur Erlösung (moksha) oder Befreiung aus dem Kreislauf der Wiedergeburten (samsara) führt. Gewaltlosigkeit impliziert hier nicht nur Vegetarismus und Vermeiden des Tötens jeder Art von Lebewesen, sondern auch Wahrhaftigkeit, Nicht-Stehlen, sexuelle $\mathrm{Zu}-$ rückhaltung und Verzicht auf unnötigen Besitz [26].

Darüber hinaus impliziert Gewaltlosigkeit aber auch die Anerkennung des Standpunkts, der Perspektive und der Interessen anderer, was in der Philosophie des Anekantavada, Nayavada und Syadvada zu einer eigenen erkenntnistheoretischen Position konzeptualisiert wurde. «An» heisst nicht oder kein, «eka» heisst ein, «anta» heisst Eigenschaft und «vada» heisst Denkschule oder Lehre; so ist «Anekantavada» also die Lehre davon, dass es nicht nur eine Eigenschaft bzw. Perspektive gibt. «Syad» heisst Bedingung oder Perspektive, und so ist «Syadvada» die Lehre von der Bedingtheit oder Perspektivität jeder Aussage. «Naya» heisst partieller Standpunkt, und so ist «Nayavada» die Lehre von den partiellen Standpunkten oder Gesichtspunkten [27].

Ein klassischer Topos in der Jain-Literatur, der diese drei Prinzipien illustrieren soll, ist die Geschichte von den vier Blinden, die tastend ein ihnen unbekanntes Tier, hier einen Elefanten, untersuchen wollen. Derjenige von ihnen, der den Rüssel untersuchte, sagte: «Das Tier gleicht einem Abflussrohr»; der ein Ohr berührte, sagte, es gleiche einem Fächer; der ein Bein umarmte, sagte, es gleiche einer Säule; der den Schwanz ergriff, sagte, es gleiche einem Seil. Jeder erfuhr und berichtete einen richtigen Aspekt des Elefanten; keiner sagte etwas Falsches, aber keiner konnte eine vollständige Beschreibung geben.

Zusammen mit der im Jainismus vertretenen Position eines erkenntnistheoretischen Realismus bedeutet diese
Bedingtheit menschlichen Erkennens durch den jeweiligen Standpunkt bzw. die jeweilige Perspektive, dass es für den irdischen Menschen keine absolute Perspektive bzw. absolute Wahrheit gibt (dies ist Allwissenden vorbehalten), andererseits aber jeder von seinem Standpunkt und seiner Perspektive aus etwas (eben dadurch nur bedingt) Wahres beitragen kann. Die praktisch-ethische Konsequenz ist unter dieser Hinsicht gerade nicht ein Kampf der Ideen und Dogmen, nach dem Motto «Entwederoder», sondern Toleranz gegenüber anderen Anschauungen und Erkenntnissen, nach dem Motto "Sowohl-als auch», eben intellektuelle oder mentale Gewaltlosigkeit. Mit dieser bereits im 6. Jahrhundert v. Chr. entwickelten Philosophie, die unter anderen auch Mahatma Gandhi (1869-1948) inspirierte, hätten - wäre sie einflussreicher geworden - viele, wenn nicht alle Glaubens- und Wissenschaftskriege verhindert werden können [28].

Hilft diese Lehre bzw. Perspektive heutigen Zeitgenossen? Gibt es nun eine Wahrheit, für die es sich zu kämpfen lohnt? Oder gibt es immer nur verschiedene Standpunkte und Perspektiven hinsichtlich einer Sache, die niemand alle einnehmen und erkennen kann, weshalb man auch niemanden kritisieren oder widerlegen, sondern vielmehr alles tolerieren bzw. sich mit allen vernetzen soll? Sollte das Thema des LMHI Homeopathic World Congress 2017 in Leipzig, "Networking in Medical Care», so gemeint gewesen sein: je mehr Vernetzung, desto besser?

\section{Perspektiven auf die Polyperspektivität}

Vergleicht man Nietzsches Umgang mit dem Phänomen der Polyperspektivität mit dem des Jainismus, so fällt auf, dass Nietzsches Ansatz, bedingt durch seine Erfahrung mit bigotten und heuchlerischen Moralvorstellungen seiner Zeitgenossen, von einem grundsätzlichen Misstrauen gegenüber philosophischen oder theologischen Allgemeinplätzen geprägt war und er seine Absage an Wahrheitsansprüche jeder Art sogar bis zu einer generellen Wissenschaftskritik ausdehnte [29]. So beschränke sich die Wissenschaft auf vermeintlich "wahre», weil abstrakte, logische und beweisbare Sätze. Demgegenüber lasse sich aber nur ein kleiner Teil des Lebens beweisen, während das, was sich beweisen lässt, für das eigentliche Leben wenig Wert habe. So bestehe das Wesen der Wissenschaft gerade darin, «vieles niemals (zu) sehen, vieles falsch (zu) sehen, vieles hinzu(zu)sehen» [30]. In dezidierter Abgrenzung von dem Genre vermeintlich logisch stringenter Traktate hinterliess Nietzsche konsequenterweise nur Aphorismen, also Gedankensplitter aus verschiedenen Perspektiven, und warnte seine Leser zudem davor, seine einzelnen Fragmente absolut zu nehmen oder sich über Widersprüchlichkeiten zu mokieren, zumal er selbst ja damit kokettierte, sich hinter Masken zu verber- 
gen, sodass keine seiner Aussagen isoliert, sondern immer nur im Kontext mit seiner jeweiligen Position und Absicht $\mathrm{zu}$ verstehen sei. Als einziges Kriterium für die Relevanz und Tauglichkeit einer Aussage galt für Nietzsche: Sie muss dem Leben dienlich sein, allerdings nicht einem unscheinbaren fremdbestimmten sklavischen, sondern einem freien, stolzen, selbstbestimmten und genussvollen Dasein.

Im Gegensatz zu Nietzsches durchtriebener polyperspektivischer Dekonstruktion von traditionellen Glaubenssätzen, die durch die gesellschaftliche Praxis in den Industriestaaten hohl und unglaubwürdig geworden waren, wirkt die Lösung des Jainismus dem Polyperspektivismus gegenüber arglos und friedfertig. Indem indischen Asketen und spirituellen Führern seit Jahrtausenden als Ziel eben nicht, wie Nietzsche, die ewige Wiederkehr eines dionysischen leiblichen Lebens vorschwebte, sondern vielmehr die Loslösung von irdischen Anhaftungen, sei es an materielle oder geistige Güter, mussten sie sich von konträren Geltungsansprüchen anderer nicht bedroht fühlen, sondern konnten auch diesen gegenüber grösste Toleranz aufbringen. Hintergrund scheint die Haltung zu sein, dass es darauf eben nicht ankomme, sondern dass es - die eigene spirituelle Entwicklung betreffend - Wichtigeres gebe.

Heutige Mediziner und Intellektuelle sind in der Regel weder Aspiranten zu dionysischen Übermenschen noch auf der Suche nach Erlösung oder Weltflucht aus dem Samsara, sodass sich für sie weder Nietzsches noch Mahávíras Modell direkt übernehmen lässt. Wie könnten aber zum Beispiel Homöopathen und ihre Kritiker mit dem real existierenden Problem der Polyperspektivität, das mittlerweile ja alle Bereiche unserer Kultur betrifft, umgehen? Muss jede Aussage jedes Apologeten und jedes Skeptikers toleriert oder gar anerkannt werden - oder ist manches einfach richtig und manches schlicht falsch, und zwar allgemeingültig und unwiderlegbar?

Im Bereich der Politik führte bekanntlich der Aufstand des Bürgertums gegen die Sonderrechte des Adels und Klerus dazu, dass spätestens seit der Französischen Revolution, also seit der Zeit Hahnemanns, demokratische Republiken als beste Staatsform angesehen wurden, in denen das Problem der Perspektiven- und Meinungsvielfalt bei Wahlen so gelöst wird, dass die Stimme jedes Einzelnen gleich gewichtet und damit egalitär anerkannt wird, ohne Rücksicht auf eventuelle Unterschiede hinsichtlich Herkunft, Vermögen, Bildung, Intelligenz, Verdienste oder Ähnliches. Das rein summarische Ergebnis entscheidet hier darüber, was allgemein gelten soll. Innerhalb des Staates wird durch das Prinzip der Gewaltenteilung bzw. der «Checks and Balances» versucht, die Macht der Legislative, Exekutive und Jurisdiktion einzugrenzen [31]. Im politischen System moderner demokratischer Staaten spielt das Kriterium wahr oder falsch also keine Rolle mehr, sondern es geht um die Durchsetzung und Umsetzung von Mehrheitsentscheidungen und die gegenseitige Kontrolle partieller Machtansprüche.

In der Wissenschaft ging man allerdings - im Abendland - bis zu Hahnemanns Zeit davon aus, dass sich anhand von logischen Schlüssen und - seit der Begründung der naturwissenschaftlichen Methode in der Neuzeit auch durch Erfahrung und Experimente die Wahrheit bzw. wahre Sätze aufstellen und Unwahrheiten widerlegen lassen. Während dies bei materiellen mechanischen Zusammenhängen etwa innerhalb der Physik oder Chemie relativ gut funktionierte und zu einem Set von Naturgesetzen führte, die den Konsens so gut wie aller Fachleute gefunden haben, war und ist das bei Lebenswissenschaften wie der Medizin sehr viel schwerer - falls überhaupt möglich. Die Geschichte der Medizin bietet eine Fülle von Beispielen, wie - nicht zuletzt zu Hahnemanns Zeit - die verschiedensten Heilsysteme um Wahrheits- und Geltungsansprüche rangen, jedes mit Berufung auf die Erfahrung, die Vernunft und die eigenen Heilerfolge [32, 33].

Mittlerweile haben sich im Zuge der Ausdifferenzierung der Einzelwissenschaften neue Fächer wie die Wissenschaftstheorie als eigene Disziplinen abgespalten, die nun selbstreflexiv die Bedingungen ihrer Möglichkeit bzw. die eigenen Voraussetzungen, Denkstile, Paradigmen und Konstrukte, aber auch ihre eigenen Grenzen und blinden Flecke untersuchen, wie etwa im Unabschliessbarkeitstheorem von Kurt Gödel (1906-1978) [34]. Wissenschaftssoziologische Untersuchungen wiederum kommen zu dem Ergebnis, dass Wissenschaft weniger als Suche nach der Wahrheit als vielmehr als soziales System zu verstehen sei, in dem ökonomische Anreize, persönliche Rivalitäten, Interessen der Industrie, aber auch institutionelle, bürokratische und technische Rahmenbedingungen die entscheidende Rolle spielen [35]. Auch in der akademischen zeitgenössischen Philosophie wird derzeit unter anderem eine Sinnfeld-Ontologie vertreten, wonach alles, was sich innerhalb eines Sinnfeldes bzw. einer bestimmten Perspektive beschreiben lässt, auch wirklich existierte (z.B. auch Einhörner auf dem Mond - wenngleich eben nur als Vorstellung). Wirklichkeit sei jedenfalls auf indefinit viele Weisen lesbar [36].

Trotz dieses wissenschaftlichen Dekonstruktionsprozesses, der zu einer allgemeinen Verunsicherung sowohl bei Wissenschaftlern als auch bei Laien geführt hat, sind manche damit zusammenhängenden gesellschaftlichen Prozesse doch durchaus zu erfassen und zu benennen, zumindest aus einer sozioökonomischen und kulturgeschichtlichen Perspektive. So gesehen scheinen aber nicht nur wissenschaftliche Ideale wie Objektivität, Neutralität oder Naturgesetzlichkeit, sondern inzwischen auch politische Errungenschaften der Aufklärung wie Freiheit, Autonomie oder Demokratie in eine schwere Krise geraten $\mathrm{zu}$ sein. Seit makroökonomische und -technologische 
Umbrüche wie Globalisierung und Digitalisierung das Leben jedes Einzelnen beherrschen, scheinen die eigentlichen Player der Weltgeschichte nicht mehr freie, autonome und kritische Individuen zu sein, sondern - neben dem Geld - Daten, Kennziffern und Algorithmen [37]. Individuen mögen zwar im subjektiven Gefühl der Freiwilligkeit zum Beispiel ihre persönlichen Daten leichtfertig dem anonymen Internet preisgeben, sich als Unternehmer ihrer eigenen Ich-AG selbst bis zur Erschöpfung ausbeuten, ihre Zeit der immer nur reaktiven Empörung über schnell hochgekochte Tagesereignisse opfern, Populisten wählen usw. - bei kühler Betrachtung lässt sich jedoch zeigen, dass das Verhalten so gut wie jedes Konsumenten den Eigentümern von Big Data bereits im Voraus bekannt und von ihnen auch manipulierbar ist [38]. Durch die strukturelle Herrschaft des Geldes, das alles, was es berührt, zu Waren verwandelt, deren Wert bezifferbar und messbar ist, wird auch das Streben von Wissenschaftlern immer mehr auf das Erreichen bestimmter Kennziffern, Evaluationsergebnisse oder Drittmittel gelenkt [39]. Gegenüber den heute real existierenden $\mathrm{Zu}$ ständen in Wissenschaft und Wirtschaft wirkt das aufklärerische Ideal autonomer, selbstbestimmter und kritischer Subjekte wie eine idealistische Illusion.

\section{Umgang mit Polyperspektivität}

Wenn aber - nach dem heutigen Stand wissenschaftlicher Erkenntnis - die Voraussetzung wissenschaftlicher Erkenntnis, nämlich freie, kritische und von sozialen, ökonomischen, politischen und anderen Zwängen unabhängige Subjekte, immer seltener anzutreffen sind: Mit wem sollte man sich dann noch - um der Wahrheitsfindung willen - vernetzen bzw. austauschen? Wenn tatsächlich jeder Gegenstand aus einer Vielzahl von Perspektiven $\mathrm{zu}$ betrachten ist, der einzelne Mensch aber nicht alles selbst untersuchen kann, wäre es doch ein Gewinn, sein Wissen mit anderen teilen zu können? Das entspräche auch ganz der klassischen Idee der «universitas magistrorum et scolarium», die seit dem 12./13. Jahrhundert in Europa als Universität ihre institutionalisierte Form annahm und gleichwohl auf dem Prinzip von «Freiheit in Forschung und Lehre» aufbaute - das heute ebenfalls extrem bedroht ist.

Sollte Polyperspektivität, um von Assoziationen wie erdrückender Pluralität oder Beliebigkeit wegzukommen, vielleicht besser mit «i» statt «y» geschrieben werden, also «Poliperspektivität»? So schreibt man zum Beispiel ja auch Poliklinik mit «i», nicht mit «y», da der Begriff hier nicht von «poly» (viel), sondern von «polis» (Stadt/Staat) kommt. Eine Poliklinik sollte ja nicht eine blosse Ansammlung beliebig vieler Kliniken unter einem Dach sein, sondern so etwas wie ein Klinikverbund, der - ähnlich wie eine Polis - zwar aus einzelnen Abteilungen besteht, jedoch ebenso ein organisches Ganzes bildet.

Wäre die griechische Polis also ein Modell für das heutige Problem, eine Vielzahl von Perspektiven in der Wissenschaft sozusagen unter einen Hut zu bekommen, d.h. einen Ausgleich, eine Integration bzw. eine wahre und gerechte Ordnung herzustellen? Woran orientierte sich aber die griechische Polis? Trotz aller wissenschaftlichen, technischen und ökonomischen Errungenschaften war das Vorbild, das Paradigma, der griechischen Polis der Götter-Olymp, wie er von Homer beschrieben und von allen Bürgern gelernt wurde. Dieser war ein abgestuftes System von Göttern, die ihre eigene Wesensart bzw. Perspektive hatten und die spontan und flexibel miteinander kommunizierten, was meist auch Auswirkungen auf die Menschen hatte.

Als Staatsphilosoph hatte auch Platon - bei aller Emphase auf die logische wissenschaftliche Durchdringung des Staatsgefüges - immer wieder auch auf die Wichtigkeit des Mythos, d.h. der grossen Erzählung, die alle Bürger, auch die Nicht-Philosophen, verbindet, hingewiesen, ebenso wie etwa auf die wichtige Rolle der Musik in der Ausbildung der Wächter, damit diese später zu tapferen Soldaten werden [40].

Es scheint, als hätte Platon noch etwas gesehen und in seine Philosophie eingebaut, was spätestens seit der Aufklärung aus ideologischen Gründen nicht mehr berücksichtigt werden konnte: dass nämlich die wissenschaftliche Vernunft, der Logos, zwar viele Aspekte einer intakten Gemeinschaft erhellen und beherrschen, ihre harmonische Funktion als Ganzheit aber nicht wirklich erfassen oder garantieren kann. Als ob der Logos nur für einen mittleren Kernbereich dieses Themas wirklich zuständig wäre und an beiden Enden, zum Allgemeinen wie zum Individuellen hin, unscharf werden muss, beanspruchte Platon weder, logisch erklären und nachvollziehen $\mathrm{zu}$ können, was eine Gemeinschaft im Innersten zusammenhält, noch, was ein Individuum zu einem tugendhaften, also tüchtigen bzw. tauglichen Individuum macht. Ein prosperierendes Staatsgebilde braucht nach Platon daher nicht nur einen rationalen Logos, sondern auch einen Mythos und ein Ethos.

Nachdem dieser Gedanke heute etwas ungewohnt erscheint, möge noch ein weiteres Beispiel für antike nachhaltige Staatskunst angeführt werden, nämlich die Konzeption des Christentums, das bekanntlich aus den beiden Wurzeln jüdischer Glaube und griechische (vor allem platonische) Philosophie besteht. Das Christentum mag zwar vordergründig als Religion des Wortes aufgefasst werden (so beginnt das Johannesevangelium mit dem Satz: «Im Anfang war das Wort», en arché én ho lógos, Joh 1,1) [6], doch werden in der Konzeption der Trinität die anderen beiden Bereiche als ebenso wichtig, ja als gleichwertige Hypostasen des einen Gottes aufge- 
fasst. So entspricht dem Sohn und seiner Verkündigung der diskursive Bereich des Logos, dem Vater der Bereich der überlieferten Schöpfungsgeschichten, also dem Mythos, und der Heilige Geist dem Bereich der frommen Haltung bzw. Tugenden, also dem Ethos. Keiner der drei Bereiche ist entbehrlich; alle drei bilden zusammen eine untrennbare Einheit. Das so konzipierte Kirchenschiff hat inzwischen den Test von zwei Jahrtausenden erfolgreich überstanden.

Diese Art von ganzheitlichem Staatsverständnis ist spätestens seit der Aufklärung, die aus heutiger kritischer Sicht eine Art Epiphänomen zeitgleicher ökonomischer Umbrüche wie Monetarisierung, Industrialisierung und Ökonomisierung des Lebens darstellt, unter die Räder gekommen. Aufklärerisch gedacht sollte fortan alles, also auch gesellschaftliches Zusammenleben, ausschliesslich rational zu lösen sein. Dass sich pure Rationalität, die letztlich ja auf dem Denken in der Geldform beruht [41], aber letztlich selbst auffrisst, hätten sich ihre damaligen Protagonisten nicht träumen lassen. Wie bereits erwähnt, wurden sukzessive zunächst der bis dahin noch relevante Bereich des Mythos sozusagen angeknabbert und ausgehöhlt, indem Religionen als Menschenwerk oder Droge für das Volk denunziert wurden; dann der Bereich des Ethos, also der Tugend, indem die Verdienste vorbildlicher trefflicher Männer etwa psychoanalytisch auf deren Neurosen oder sonstige niedere Antriebe zurückgeführt wurden. Schliesslich nahm sich die Wissenschaft der Postmoderne und des Dekonstruktivismus sich selber vor, um sich selbst den Ast abzusägen, auf dem sie sass.

Die politischen, ökonomischen und sozialen Folgen bzw. Verwerfungen dieser Selbst-Entlegitimierung der Wissenschaft sind alarmierend und unübersehbar. Sind erst einmal (fast) alle Autoritäten, grossen Erzählungen, Respektspersonen und wissenschaftlichen Theorien dekonstruiert und damit relativiert oder gar irrelevant geworden, lässt sich im Zeitalter der Postfaktizität willkürlich mit sogenannten «alternativen Wahrheiten» durchregieren, Stimmung machen und Manipulation orientierungsloser Subjekte betreiben. Aus der Unzahl nivellierter Daten der Wissenschaften können sich Demagogen oder Terroristen selektiv einzelne Perspektiven herausgreifen und damit einen neuen Populismus oder Fundamentalismus begründen, so als hätte es noch nie eine Aufklärung gegeben. Da die rationalistischen, immer noch auf das Paradigma autonomer Subjekte und demokratischer Volkssouveränität fixierten Geisteswissenschaften für die anderen, vergessenen Dimensionen menschlicher Interaktionen (neben Logos eben Mythos und Ethos) keine erschliessenden Kategorien haben, können sie den neueren Entwicklungen so gut wie nichts entgegenhalten.

\section{Die Perspektiven Samuel Hahnemanns}

In dieses makroskopische Bild einer postfaktischen, postdemokratischen und postaufklärerischen Gesamtsituation fügt sich nahtlos die Tatsache ein, dass es auch auf der Mikroebene wissenschaftlicher Auseinandersetzungen offenbar einer nicht unerheblichen Anzahl von Playern möglich ist, wissenschaftliche Forschungen und Studienergebnisse schlicht nicht zur Kenntnis zu nehmen. Wenn zum Beispiel wissenschaftlich nachgewiesen wurde, dass über $90 \%$ der Homöopathie-Studien ignoriert werden müssen, um das Urteil aufrecht zu erhalten, Homöopathie sei unwirksam [42], und wenn aber gleichzeitig Wissenschaftler und Journalisten gerade dies tun, so scheint hier die rationale Wissenschaft, der reine Logos, an seine Grenzen geraten zu sein. Wenngleich man diese Situation durchaus geisteswissenschaftlich, nämlich wissenschaftssoziologisch, sozioökonomisch, kommunikationstheoretisch, medienpolitisch usw. untersuchen und beschreiben kann, sind auch diese Erkenntnisse und Perspektiven ein zahnloser Tiger, solange hier Kräfte am Werke sind, denen es um anderes als um Wissenschaft geht.

Was könnten Homöopathen also tun - angesichts der heutigen Polyperspektivität, die innerhalb ihrer eigenen Reihen und innerhalb eines geordneten wissenschaftlichen Rahmens durchaus fruchtbar sein kann, die aber, wenn sie ausufert und alle Bereiche des gesellschaftlichen Lebens erfasst, kontraproduktiv wird und einer neuen Willkür Tür und Tor öffnet? Ist die Homöopathie, als Produkt der Zeit der Aufklärung, vielleicht sogar mit ein Teil des seitdem verschärften Problems?

Hahnemann lebte in der Zeit der Aufklärung, doch war er kein aufklärerischer Fundamentalist. Zwar betonte er in seiner Frühzeit - in methodischer Hinsicht - die Rationalität seiner Heilkunde, vor allem in der 1. Auflage seines «Organon der rationellen Heilkunde» (1810), doch tilgte er den dort $20 \mathrm{Mal}$ vorkommenden Begriff «rationell» bereits in der 2. Auflage von 1819 komplett aus diesem Werk (mit einer Ausnahme, wo er dazu benutzt wurde, die Fehler der alten «rationellen» Schule anzuprangern), das er fortan «Organon der Heilkunst» nannte [43]. Wie auch andere gebildete Kollegen thematisierte Hahnemann in seinen umfangreichen Publikationen aber nicht nur medizinische Inhalte, sondern beteiligte sich auch eifrig am allgemeinen Diskurs über weltanschauliche, philosophisch-theologische und ethische Fragen, was ihn selbst als rechtschaffenen, gläubigen und wohlwollenden Bürger zur Erscheinung brachte und konstituierte.

Konkret wurde Hahnemann nicht müde, Gott als den gütigen und weisen Schöpfer der Menschen zu preisen und die Endlichkeit des menschlichen Erkenntnisvermögens zu betonen, aber auch die hohe Bestimmung des Menschen, sich «auf der Leiter beseligender Empfindun- 
gen, menschenveredelnder Tätigkeiten und weltendurchschauender Kenntnisse» «dem grossen Urgeist zu nähern» [44]. Insgesamt - und das ist der entscheidende Unterschied zu aufklärerischen Fundamentalisten oder postmodernen Agnostikern - präsentierte sich Hahnemann nicht nur als rationaler Fachwissenschaftler, sondern ebenso als verantwortungsvoller Staatsbürger, dem es ein Anliegen war, sowohl den religiösen Überbau (letztlich den kulturtragenden Mythos bzw. das grosse Narrativ) als auch die Wertschätzung der Sittlichkeit (den Bereich des Ethos) aufrechtzuerhalten und publizistisch seinen Teil dazu beizutragen. Dass in den Bereichen an beiden Enden der Vernunft, wo also, wie erwähnt, der Logos von seinem Kernbereich zum Mythos und zum Ethos übergeht, nicht rein rational argumentiert werden kann, war für Hahnemann, der auch die Klaviatur der Rhetorik und Polemik beherrschte, kein Problem.

\section{Hahnemanns Auftrag}

Unter dieser neu erschlossenen ganzheitlichen Perspektive ist nun Hahnemanns Imperativ von 1817, der seit 200 Jahren zwar von vielen zitiert, aber kaum wirklich verstanden wurde, neu zu bedenken und auszulegen.

Hahnemann sagte «Macht's nach», was sich zweifellos auf das Offensichtliche, nämlich das Praktizieren seiner Heilmethode bezog. Hahnemann fordert keine Loyalität oder Rücksichtnahme auf seine Person, sondern ein Nachmachen dessen, was er selbst vormachte. Was aber machte Hahnemann nicht alles? Ein oberflächliches Verständnis wird hier bei der Tatsache, dass Hahnemann Homöopathie praktizierte, hängenbleiben und sich damit begnügen. Bei genauerem Hinsehen bzw. Lesen seines umfangreichen literarischen Vermächtnisses wird man allerdings gewahr, dass Hahnemann die von ihm gefundene homöopathische Methode immer sozusagen auch metaphysisch-narrativ in einen grösseren sittlichen $\mathrm{Zu}$ sammenhang einordnete. Aus 200-jähriger Entfernung können wir Heutige, die bis vor Kurzem - verleitet vom inzwischen postfaktisch überholten Wissenschaftspositivismus - bei Hahnemanns Auslassungen über den Schöpfer, seine Mitmenschen und die Sittlichkeit noch die Nase gerümpft haben, nur über die Besonnenheit staunen, mit der Hahnemann - trotz aller rationalistischen Aufklärerei - jederzeit an der Wichtigkeit auch der Bereiche Mythos und Ethos festhielt.

Der Satz Hahnemanns an seine Rezensenten wäre damit folgendermassen dreifach auszulegen:

(1) «Macht's nach»: Praktiziert die Homöopathie bzw. Medizin so wie ich; wendet die Vorschriften an, wie ich sie im Organon verzeichnet habe! (Logos)

(2) «Macht's genau nach»: Nehmt genau wahr, was ich eigentlich alles tue, wie ich wirke, wie ich die Homöo- pathie im Kontext und im Rahmen der Endlichkeit, Bedürftigkeit, aber auch der hohen Bestimmung des Menschen zum Wohl meiner Mitmenschen praktiziere, erforsche und weiterentwickle! (Mythos)

(3) «Macht's sorgfältig nach»: Bedenkt auch die Dimension der Sittlichkeit bzw. des Ethos, denn die Praxis der Homöopathie steht und fällt mit der Sorgfalt, allgemein mit der Tugend der sie Praktizierenden, etwa mit ihrer Genauigkeit, Ehrlichkeit, Zuverlässigkeit usw.! (Ethos)

Dass für Hahnemann die Bereiche Mythos, Logos und Ethos untrennbar zusammengehörten und ihm, zumal als Freimaurer, die Dreiheit als gängiger Schlüssel der Auslegung geläufig war, möge die Stelle aus seinem Organon von 1810 belegen, wo er davor warnt, «dass Indolenz, Gemächlichkeit und Starrsinn vom Dienst am Altare der Wahrheit ausschliesst, und nur Unbefangenheit und unermüdeter Eifer zur heiligsten aller menschlichen Arbeiten (be)fähigt, zur Ausübung der wahren Heilkunde. Der Heilkünstler in diesem Geiste aber schliesst sich unmittelbar an die Gottheit, an den Weltenschöpfer an, dessen Menschen er erhalten hilft, und dessen Beifall sein Herz dreimal beseligt» [2, S. III-IV].

Auf die erläuterte Weise aufgefasst, könnte Hahnemanns Ausspruch heute, 200 Jahre nach seiner Erstpublikation, seinen verwirrten Nachfolgern wieder Orientierung und einen Hinweis geben, wo Ursache und Schlüssel für die mittlerweile überhand nehmende Polyperspektivität, nicht nur die Homöopathie betreffend, zu finden sind.

Während eine gewisse Polyperspektivität in Massen wissenschaftlich fruchtbar und evolutionär sogar ein Überlebensvorteil ist, wird sie im Übermass, wenn sie kontextvergessen und unkontrolliert ausufert, kontraproduktiv. Wird Polyperspektivität etwa durch Dekonstruktionen vermehrt, die auf Kosten der grossen kulturtragenden und sinnstiftenden Narrative gehen, so wäre dies als übergriffige, unseriöse Wissenschaft zu kritisieren. Das Gleiche gilt für eine inflationäre und substanzlose Vermehrung der Perspektiven durch ethisch zweifelhafte und fachlich unqualifizierte Autoren und sonstige Multiplikatoren, wie sie vermehrt in Massenmedien, vor allem in Blogs und Talkshows, anzutreffen sind.

In Abgrenzung von der allgegenwärtigen Verflachung und Simplifizierung der Berichterstattung sollte es die Homöopathenschaft also nicht dabei belassen, gute klinische Praxis zu betreiben, sondern parallel dazu sowohl an einem Gemeinschaft stiftenden Narrativ ihrer gegenwärtigen kulturgeschichtlichen Lage und Mission arbeiten als auch auf ihre Tugenden und ihren ethischen Code grössten Wert legen. Ein neues Bewusstsein für kulturelle und ethische Verantwortung wäre freilich auch vonseiten ihrer Kritiker zu begrüssen, die seit jeher dazu neigen, fixiert auf einen einzelnen (vermeintlich unlogischen) Teilas- 
pekt das Kind mit dem Bade auszuschütten. Gerade in Zeiten, wo die Rationalität bei der Lösung ihrer eigenen hausgemachten Probleme ans Ende ihres Lateins gekommen ist, dürften die schwer vernachlässigten Dimensionen Mythos und Ethos wieder an Bedeutung gewinnen.

\section{Disclosure Statement}

Kein Interessenkonflikt.

\section{Literatur}

1 Hahnemann S: Reine Arzneimittellehre. Band 3. Dresden, Arnold, 1817, p V; in Schmidt JM, Kaiser D (Hrsg): Samuel Hahnemann. Gesammelte kleine Schriften. Heidelberg, Haug, 2001, p 675.

2 Hahnemann S: Organon der rationellen Heilkunde. Dresden, Arnold, 1810.

3 Hahnemann S: Fragmenta de viribus medicamentorum positivis sive in sano corpore humano observatis. 2 Bände. Leipzig, Barth, 1805

4 Hahnemann S: Reine Arzneimittellehre. 6 Bän de. Dresden, Arnold, 1811-1821.

5 Schmidt JM, Kaiser D (Hrsg): Samuel Hahnemann. Gesammelte kleine Schriften. Heidelberg, Haug, 2001.

6 Nestle E, Aland K (Hrsg): Novum Testamentum Graece, ed 26. Stuttgart, Deutsche Bibelgesellschaft, 1983.

7 Paracelsus: Das Buch Paragranum (1530); in Peuckert WE (Hrsg): Theophrastus Paracelus Werke. Band 1. Medizinische Schriften. Darmstadt, Wissenschaftliche Buchgesellschaft, 1990, p 501.

8 Schmidt JM: Kompaktwissen Homöopathie Grundlagen, Methodik und Geschichte. Schriften zur Geschichte und Theorie der Homöopathie. Band 2. München, bge, 2016.

9 Geiselberger H (Hrsg): Die große Regression. Eine internationale Debatte über die geistige Situation der Zeit. Berlin, Suhrkamp, 2017.

10 Weymar C, Heißmann N: Die HomöopathieLüge. München, Piper, 2012.

11 Aust N: In Sachen Homöopathie. Eine Be weisaufnahme. Ebersdorf, 1-2-Buch, 2013.

12 Attard P: The stability of nanobubbles. Eur Phys J Special Topics 2013;1-22.

13 Demangeat JL: Nanosized solvent superstructures in ultramolecular aqueous dilutions: twenty years' research using water proton NMR relaxation. Homeopathy 2013;102:87-105.

14 Elia V, Ausanio G, Gentile F, Germano R, Napoli E, Niccoli M: Experimental evidence of stable water nanostructures in extremely dilute solutions, at standard pressure and temperature. Homeopathy 2014;103:44-50.

15 Demangeat JL: Gas nanobubbles and aqueous nanostructures: the crucial role of dynamization. Homeopathy 2015;104:101-115.
16 Oberbaum M, Frass M, Gropp C: Unequal brothers: are homeopathy and hormesis linked? Homeopathy 2015;104:97-100.

17 Oberbaum M, Gropp C: Update on hormesis and its relation to homeopathy. Homeopathy 2015;104:227-233.

18 Dei A, Bernadini S: Hormetic effects of extremely diluted solutions on gene expression. Homeopathy 2015;104:116-122.

19 Olioso D, Marzotto M, Bonafini C, Brizzi M, Bellavite P: Arnica montana effects on gene expression in a human macrophage cell line. Evaluation by quantitative Real-Time PCR Homeopathy 2016;105:131-147.

20 Khuda-Bukhsh AR, Sikdar S: Condurango $30 \mathrm{C}$ induces epigenetic modification of lung cancer-specific tumour suppressor genes via demethylation. Forsch Komplementmed 2015;22:172-179.

21 Tournier AL, Roberts ER: Cutting Edge Research in Homeopathy: HRI's second international research conference in Rome. Homeopathy 2016;105:3-7.

22 von Ammon K, Baumgartner S, Behnke J, Frei-Erb M, Kösters C, Teut M, Torchetti L: Der aktuelle Stand der Forschung zur Homöopathie. Köthen, WissHom, 2016.

23 Schmidt JM: Die Entstehung, Verbreitung und Entwicklung von Heilsystemen als Gegenstand der Medizingeschichte - am Beispiel der Homöopathie (2007); in Schmidt IM: Schriften zur Geschichte und Theorie der Homöopathie. Band 5. München, bge, 2016, 39-65.

24 Assmus J: Exodus. Die Revolution der Alten Welt. München, Beck, 2015.

25 Nietzsche F: Die fröhliche Wissenschaft. Aph. 125, vgl. 108, 343; in Nietzsche F: Sämtliche Werke. Kritische Studienausgabe in 15 Bänden. Band 3. München, dtv, 1980.

26 Chakravarti A: The Religion of Ahimsa. The Essence of Jaina Philosophy and Ethics. Chennai, Varthamanan Pathipagam, 2005.

27 Shah N: Jainism. The World of Conquerors. 2 volumes. Brighton, Sussex Academic Press, 1998.

28 Zydenbos RJ: Jainism Today and Its Future. München, Manya, 2006.
29 Babich B: Nietzsches Wissenschaftsphilosophie; in Hermand J (Hrsg): German Life and Civilization. Vol. 52. Oxford, Peter Lang, 2011.

30 Nietzsche F: Nachgelassene Fragmente. November 1887-März 1888; in Colli G, Montinari M (Hrsg): Friedrich Nietzsche. Sämtliche Werke. Kritische Studienausgabe. München, dtv, 1980, Band 13, p 193.

31 Winkler HA: Geschichte des Westens. Von den Anfängen in der Antike bis zum 20. Jahrhundert. München, Beck, 2013.

32 Leibbrand W: Heilkunde. Eine Problemgeschichte der Medizin. Freiburg, Karl Alber, 1953.

33 Rothschuh KE: Konzepte der Medizin in Vergangenheit und Gegenwart. Stuttgart, Hippokrates, 1978.

34 Seiffert H, Radnitzky G: Handlexikon der Wissenschaftstheorie. München, dtv, 1994.

35 Weingart P: Wissenschaftssoziologie. Bielefeld, transcript, 2003.

36 Gabriel M: Warum es die Welt nicht gibt. Berlin, Ullstein, 2016.

37 Schirrmacher F: Ego. Das Spiel des Lebens. München, Blessing, 2013.

38 Han BC: Psychopolitik. Neoliberalismus und die neuen Machttechniken. Frankfurt am Main, Fischer, 2016.

39 Crouch C: Die bezifferte Welt. Wie die Logik der Finanzmärkte das Wissen bedroht. Berlin, Suhrkamp, 2017.

40 Platon: Politeia; in Burnet I (Hrsg): Platonis Opera. Band 4. London, Oxford University Press, 1982.

41 Brodbeck KH: Die Herrschaft des Geldes. Geschichte und Systematik. Darmstadt, Wissenschaftliche Buchgesellschaft, 2009.

42 Hahn RG: Homeopathy: meta-analyses of pooled clinical data. Forsch Komplementmed 2013;20:376-381.

43 Schmidt JM: Grundlagenwerk: Das «Organon der rationellen Heilkunde»; in Schmidt JM: Schriften zur Geschichte und Theorie der Homöopathie. Band 5. München, bge, 2016, 129-138.

44 Hahnemann S: Aeskulap auf der Wagschale (1805); in Schmidt JM, Kaiser D (Hrsg): Samuel Hahnemann. Gesammelte kleine Schriften. Heidelberg, Haug, 2001, p 370. 